\title{
Polynomial time quantum algorithms for certain bivariate hidden polynomial problems
}

\author{
Thomas Decker* $\quad$ Peter Høyer ${ }^{\dagger} \quad$ Gábor Ivanyos ${ }^{\ddagger} \quad$ Miklos Santha $^{\S}$
}

October 9, 2013

\begin{abstract}
We present a new method for solving the hidden polynomial graph problem (HPGP) which is a special case of the hidden polynomial problem (HPP). The new approach yields an efficient quantum algorithm for the bivariate HPGP even when the input consists of several level set superpositions, a more difficult version of the problem than the one where the input is given by an oracle. For constant degree, the algorithm is polylogarithmic in the size of the base field. We also apply the results to give an efficient quantum algorithm for the oracle version of the HPP for an interesting family of bivariate hidden functions. This family includes diagonal quadratic forms and elliptic curves.
\end{abstract}

\section{Introduction}

In the hidden polynomial problem (HPP) we are given an oracle for a function of the form $\mathcal{E}\left(F\left(x_{1}, \ldots, x_{n}\right)\right)$, where $F$ is an unknown polynomial in $n$ variables of degree at most $D$ over the finite field $\mathbb{F}$ and where $\mathcal{E}$ is an unknown unique encoding of elements of $\mathbb{F}$ by binary strings. This means that the level sets of the oracle coincide with the level sets of the polynomial. The task is to determine the polynomial $F$. Obviously, $F$ can only be determined up to a constant additive term and up to another constant multiplicative factor. Therefore, we consider polynomials with fixed constant term (usually zero) and in which another monomial is fixed (usually it has coefficient 1). In the quantum setting, the oracle is actually a unitary transformation which maps states of the form

$$
\left|x_{1}\right\rangle\left|x_{2}\right\rangle \cdots\left|x_{n}\right\rangle|0\rangle \quad \text { to } \quad\left|x_{1}\right\rangle\left|x_{2}\right\rangle \cdots\left|x_{n}\right\rangle\left|\mathcal{E}\left(F\left(x_{1}, \ldots, x_{n}\right)\right)\right\rangle \text {. }
$$

\footnotetext{
*Centre for Quantum Technologies, National University of Singapore, Singapore 117543 (t.d3ck3r@gmail.com).

$\dagger$ Department of Computer Science and Institute for Quantum Science and Technology, University of Calgary, 2500 University Drive N.W., Calgary, Alberta, Canada, T2N 1N4 (hoyer@ucalgary.ca).

${ }^{\ddagger}$ Institute for Computer Science and Control, Hungarian Academy of Sciences, Budapest, Hungary (Gabor.Ivanyos@sztaki.mta.hu).

$\S$ LIAFA, Univ. Paris 7, CNRS, 75205 Paris, France; and Centre for Quantum Technologies, National University of Singapore, Singapore 117543 (miklos.santha@liafa.jussieu.fr).
} 
We measure the complexity in terms of the number of bits that are necessary to describe the polynomial $F$, which is $\Omega(\log |\mathbb{F}|)$ if $n$ and $D$ are constant. We say an algorithm is efficient if its time complexity is polynomial in $\log |\mathbb{F}|$. We assume that each oracle query can be conducted in one time step, where needed.

The HPP was introduced by Childs, Schulman and Vazirani [1 in an attempt to generalize the study of properties of algebraic sets hidden by black-box functions from linear structures, instantiated by the well-known hidden subgroup problem (HSP), to higher degree cases. They showed that when the degree of the hidden polynomial as well as the number of variables is constant, a typical polynomial can be determined by a polynomial number of queries. Decker, Ivanyos, Santha and Wocjan [2] designed an efficient quantum algorithm for the HPP for multivariate quadratic polynomials over fields of constant characteristic. In [3], Decker, Draisma and Wocjan considered the hidden polynomial graph problem (HPGP), a special case of the HPP where $F\left(x_{1}, \ldots, x_{n}\right)$ is of the form $x_{n}-f\left(x_{1}, \ldots, x_{n-1}\right)$ for some polynomial $f\left(x_{1}, \ldots, x_{n-1}\right)$. They showed how to reduce the HPGP, when $n$ and the degree are constant, to the bivariate case, that is to the case of a hidden polynomial $F(x, y)$ of the form $y-f(x)$. They also gave an efficient quantum algorithm for the bivariate case when the degree of $f$ is a constant and smaller than the characteristic of the base field $\mathbb{F}$. The algorithm of [3] used a technique analogous to the pretty good measurement framework of [4] for solving the HSP in certain semidirect product groups.

An explanation for why these two problems can be solved with very similar tools was given in [2] where it was proven that the bivariate HPGP can be efficiently reduced to a special instance of the HSP. In fact over prime fields this coincides with the problem considered in [4]. Interestingly, there is (almost) a reduction in the other direction as well: based on [4], it is shown in [2] that the hidden subgroup problem in $\mathbb{Z}_{p}^{m} \rtimes \mathbb{Z}_{p}$ can be efficiently reduced to a multidimensional version of the HPGP.

In this paper we propose a novel approach for solving a slightly more difficult version of the bivariate HPGP in which, rather than an oracle, we are given quantum states as input. To be more specific, the input consists of several level set superpositions of the function $F(u, x)=u-f(x)$, that is, quantum states ${ }^{1}$ of the form

$$
\sum_{x \in \mathbb{F}}|w+f(x)\rangle|x\rangle
$$

where each state comes with an unknown and possibly different element $w \in \mathbb{F}$. In the following, we do not assume anything on the various $w$ corresponding to different input states. This definition of the HPGP is more general than the oracle version, because from the oracle we can easily obtain the level set superpositions of Eq. (1) for random $w$ according to a distribution reflecting the frequency of $w$ appearing as a value of $F$. Our main result is the following.

Theorem 1. Let $D$ be a constant and let $f(x)=\sum_{s=1}^{D} \nu_{s} x^{s} \in \mathbb{F}[x]$. Then there is a quantum algorithm which, given $O(1)$ states of the form (1) for various and unknown $w \in \mathbb{F}$, determines the hidden coefficients $\nu_{1}, \ldots, \nu_{D}$ efficiently.

Actually, the special case of Theorem 1 where the characteristic of $\mathbb{F}$ is greater than $D$ could also be proved using the method of [3], because it is also a quantum algorithm that

\footnotetext{
${ }^{1}$ In order to simplify notation, we omit the normalization factors of state vectors in Section 1 and 2
} 
works on states of the form (1). Our result is nonetheless new for small characteristics. Observe that the HSP is only discussed in the oracle setting and cannot directly be applied to the states (1).

As an application, we present an efficient quantum algorithm which solves the oracle version of the HPP for the family of bivariate hidden functions of the form $F(x, y)=$ $g(y)-f(x)$ where $g(y)$ is a fixed and known non-constant polynomial of degree $D^{\prime}$ in $y$ and $f(x)$ is an unknown polynomial of degree at most $D$ in $x$ with fixed constant term. This class includes polynomials of the form $y^{2}-\nu x^{2}$ (diagonal quadratic forms) as well as those of the form $y^{2}-f(x)$ where $f(x)$ is of degree 3 or higher (elliptic and hyperelliptic curves). In contrast to Theorem 1, where the error can be made arbitrarily small, the algorithm for this theorem has an ingredient which gives the correct result with a probability that is bounded by a (small) constant. Hence, we need the oracle to test the correctness of results. We show the following.

Theorem 2. Let $D$ and $D^{\prime}$ be constants and let $g(y)=\sum_{s=0}^{D^{\prime}} \mu_{s} y^{s}$ be a fixed polynomial. Furthermore, let $f(x)=\sum_{s=1}^{D} \nu_{s} x^{s}$ be a polynomial of degree $D$ with unknown coefficients $\nu_{s}$. Then, given a quantum oracle that maps states of the form $|y\rangle|x\rangle|0\rangle$ to $|y\rangle|x\rangle|\mathcal{E}(g(y)-f(x))\rangle$, the unknown coefficients $\nu_{1}, \ldots, \nu_{D}$ can be determined in polynomial time.

The proof of Theorem 1 and our method for solving the HPGP are presented in Section 2, The proof of Theorem 2 is given in Section 3.

\section{The phase linearization approach}

The high level description of our algorithm, that we call phase linearization is actually quite simple. The QFT applied to a level set superposition results in a superposition where the phases have not only linear but also higher degree exponents. Our main goal is to eliminate these non-linear exponents, once it is done the inverse QFT yields a linear equation in the unknown coefficients. To achieve this we will combine several copies of the level set superposition. The acquired freedom in the composed phase can be used, with the help of an additional register in uniform superposition, to make the exponents linear.

The elimination of the higher degree exponents will be done recursively. For this it will be convenient to consider a technical generalization of the HPGP that is suitable for recursion. However, before formulating that, we demonstrate phase linearization in the quadratic case and we also briefly outline an extension to the cubic case.

\subsection{The quadratic and cubic cases}

In this subsection, we assume that $|\mathbb{F}|$ is odd and that our input consists of several states of the form (1) where $f(x)=\nu x+\mu x^{2}$, that is we have states

$$
\sum_{x \in \mathbb{F}}\left|w+\nu x+\mu x^{2}\right\rangle|x\rangle
$$


with various unknown $w \in \mathbb{F}$. The task is to determine the coefficients $\nu$ and $\mu$. We apply to the first register the quantum Fourier transform of the field $\mathbb{F}$. This is the unitary transform, introduced in [5], that maps states $|a\rangle, a \in \mathbb{F}$, to

$$
\sum_{b \in \mathbb{F}} \omega^{\operatorname{Tr}(a b)}|b\rangle
$$

where $\operatorname{Tr}$ is the trace map from $\mathbb{F}$ to its prime field $\mathbb{F}_{p}$ and $\omega=e^{2 \pi i / p}$ is a $p$ th root of unity. Here, $p$ is the characteristic of $\mathbb{F}$, that is, $p$ is the prime for which $|\mathbb{F}|=p^{\alpha}$ with a positive integer $\alpha$. Then for an element $a \in \mathbb{F}$ the trace is $\operatorname{Tr}(a)=\sum_{j=0}^{\alpha-1} a^{p^{j}}$. A polynomial time approximate implementation of the Fourier transform of $\mathbb{F}$ is given in [5]. Here and in the following, we ignore the error coming from this approximation, because it can be made arbitrarily small with only a small overhead. This transform maps our state (2) to

$$
\sum_{y \in \mathbb{F}} \omega^{\operatorname{Tr}(y w)} \sum_{x \in \mathbb{F}} \omega^{\operatorname{Tr}\left(y \nu x+y \mu x^{2}\right)}|y\rangle|x\rangle .
$$

We measure the first register and obtain the following state (up to a global phase, which we omit)

$$
\sum_{x \in \mathbb{F}} \omega^{\operatorname{Tr}\left(y \nu x+y \mu x^{2}\right)}|x\rangle
$$

with uniformly random $y \in \mathbb{F}$. If the term $y \mu x^{2}$ were missing from the exponent in the coefficient of $|x\rangle$ in the state (3), then $y \nu$ and also $\nu$ could be obtained by applying the inverse Fourier transform of $\mathbb{F}$.

Motivated by this observation, our goal is to eliminate the quadratic term from the exponent. To this end, we take three states of the form (2) with possibly different values $w$ and we apply the Fourier transform and the measurement independently to them. In principle, we could also consider two states, but taking three states allows us to apply directly the results of [6] and [7] to simplify the presentation. In more detail, we start with the product state

$$
\sum_{x_{1}, x_{2}, x_{3} \in \mathbb{F}}\left|w_{1}+\nu x_{1}+\mu x_{1}^{2}\right\rangle\left|w_{2}+\nu x_{2}+\mu x_{2}^{2}\right\rangle\left|w_{3}+\nu x_{3}+\mu x_{3}^{2}\right\rangle\left|x_{1}, x_{2}, x_{3}\right\rangle
$$

and the result is the state

$$
\sum_{x_{1}, x_{2}, x_{3} \in \mathbb{F}} \omega^{\operatorname{Tr}\left(\nu\left(y_{1} x_{1}+y_{2} x_{2}+y_{3} x_{3}\right)+\mu\left(y_{1} x_{1}^{2}+y_{2} x_{2}^{2}+y_{3} x_{3}^{2}\right)\right)}\left|x_{1}, x_{2}, x_{3}\right\rangle
$$

for uniformly random $y_{1}, y_{2}, y_{3} \in \mathbb{F}$, which are known to us as a result of the measurements. For brevity, we write this state as

$$
\sum_{x_{1}, x_{2}, x_{3} \in \mathbb{F}} \omega^{\operatorname{Tr}\left(e\left(x_{1}, x_{2}, x_{3}\right)\right)}\left|x_{1}, x_{2}, x_{3}\right\rangle
$$

where

$$
e\left(x_{1}, x_{2}, x_{3}\right)=\nu\left(y_{1} x_{1}+y_{2} x_{2}+y_{3} x_{3}\right)+\mu\left(y_{1} x_{1}^{2}+y_{2} x_{2}^{2}+y_{3} x_{3}^{2}\right) .
$$


We abort if any of $y_{1}, y_{2}, y_{3}$ happens to be zero. If none of them is zero, we produce the superposition $\sqrt{1 /|\mathbb{F}|} \sum_{x \in \mathbb{F}}|x\rangle$ in a fourth register. The result is

$$
\sum_{x_{1}, x_{2}, x_{3}, x \in \mathbb{F}} \omega^{\operatorname{Tr}\left(e\left(x_{1}, x_{2}, x_{3}\right)\right)}\left|x_{1}, x_{2}, x_{3}\right\rangle|x\rangle .
$$

Then, with appropriately chosen elements $\delta_{1}, \delta_{2}, \delta_{3}$ (see below), we subtract $\delta_{i} x$ from the $i$ th register for $i=1,2,3$. The result is

$$
\sum_{x_{1}, x_{2}, x_{3}, x \in \mathbb{F}} \omega^{\operatorname{Tr}\left(e\left(x_{1}, x_{2}, x_{3}\right)\right)}\left|x_{1}-\delta_{1} x, x_{2}-\delta_{2} x, x_{3}-\delta_{3} x\right\rangle|x\rangle,
$$

which is in turn equal to the state

$$
\sum_{x_{1}, x_{2}, x_{3}, x \in \mathbb{F}} \omega^{\operatorname{Tr}\left(e^{\prime}\left(x_{1}, x_{2}, x_{3}\right)\right)}\left|x_{1}, x_{2}, x_{3}\right\rangle|x\rangle
$$

with

$$
\begin{aligned}
e^{\prime}\left(x_{1}, x_{2}, x_{3}\right)= & e\left(x_{1}+\delta_{1} x, x_{2}+\delta_{2} x, x_{3}+\delta_{3} x\right) \\
= & \nu\left[y_{1}\left(x_{1}+\delta_{1} x\right)+y_{2}\left(x_{2}+\delta_{2} x\right)+y_{3}\left(x_{3}+\delta_{3} x\right)\right] \\
& +\mu\left[y_{1}\left(x_{1}+\delta_{1} x\right)^{2}+y_{2}\left(x_{2}+\delta_{2} x\right)^{2}+y_{3}\left(x_{3}+\delta_{3} x\right)^{2}\right] \\
= & \nu a\left(x_{1}, x_{2}, x_{3}\right)+\mu b\left(x_{1}, x_{2}, x_{3}\right)+\nu c x+\mu d\left(x_{1}, x_{2}, x_{3}\right) x \\
& +\mu Q x^{2}
\end{aligned}
$$

where

$$
\begin{aligned}
a\left(x_{1}, x_{2}, x_{3}\right) & =y_{1} x_{1}+y_{2} x_{2}+y_{3} x_{3} \\
b\left(x_{1}, x_{2}, x_{3}\right) & =y_{1} x_{1}^{2}+y_{2} x_{2}^{2}+y_{3} x_{3}^{2} \\
c & =y_{1} \delta_{1}+y_{2} \delta_{2}+y_{3} \delta_{3} \\
d\left(x_{1}, x_{2}, x_{3}\right) & =2\left(y_{1} \delta_{1} x_{1}+y_{2} \delta_{2} x_{2}+y_{3} \delta_{3} x_{3}\right) \\
Q & =y_{1} \delta_{1}^{2}+y_{2} \delta_{2}^{2}+y_{3} \delta_{3}^{2}
\end{aligned}
$$

We choose $\delta_{1}, \delta_{2}, \delta_{3}$ in such a way that the exponent of the coefficient of $\left|x_{1}, x_{2}, x_{3}\right\rangle|x\rangle$ will become linear in $x$ for every $x_{1}, x_{2}, x_{3}$. That is, we want to have $Q=0$. Using the Las Vegas method of [6] or the deterministic algorithm of [7], we can find in time polylog $(|\mathbb{F}|)$ three elements $\delta_{1}, \delta_{2}, \delta_{3}$, that are not all zero, such that $y_{1} \delta_{1}^{2}+y_{2} \delta_{2}^{2}+y_{3} \delta_{3}^{2}=0$. Then the state we have equals

$$
\sum_{x_{1}, x_{2}, x_{3}, x \in \mathbb{F}} \omega^{\operatorname{Tr}\left(\nu a\left(x_{1}, x_{2}, x_{3}\right)+\mu b\left(x_{1}, x_{2}, x_{3}\right)+\nu c x+\mu d\left(x_{1}, x_{2}, x_{3}\right) x\right)}\left|x_{1}, x_{2}, x_{3}\right\rangle|x\rangle .
$$

We measure the first three registers. Then we obtain the state

$$
\omega^{\operatorname{Tr}(\nu a+\mu b)} \sum_{x \in \mathbb{F}} \omega^{\operatorname{Tr}(\nu c x+\mu d x)}|x\rangle,
$$

where $a=a\left(x_{1}, x_{2}, x_{3}\right), b=b\left(x_{1}, x_{2}, x_{3}\right)$, and $d=d\left(x_{1}, x_{2}, x_{3}\right)$ for uniformly random $x_{1}, x_{2}, x_{3} \in \mathbb{F}$. We abort if $d$ becomes zero. Note that $d$ is linear in $x_{1}, x_{2}, x_{3}$ and that 
none of the $y_{i}$ are zero and that not all of the $\delta_{i}$ are zero. Hence, $d$ is only zero with probability $1 /|\mathbb{F}|$. Observe that here we use the assumption that the characteristic of $\mathbb{F}$ is odd, otherwise $d$ would always be zero by definition. If $d$ is nonzero, we apply the inverse Fourier transform of $\mathbb{F}$ and obtain the state $|\nu c+\mu d\rangle$ up to some phase. After a measurement, we find the value $g=\nu c+\mu d$.

This way, we obtain a proper linear constraint (as $d \neq 0$ ) for the unknown parameters $\mu$ and $\nu$, because the values $c, d$ and $g$ are known to us. The probability that the procedure goes through is at least $(1-1 /|\mathbb{F}|)^{4}$. The cases when it is aborted are the cases when one of the values $y_{1}, y_{2}$ or $y_{3}$ is zero or when $d$ becomes zero.

As $d$ is nonzero, we can substitute $\frac{g}{d}-\frac{c}{d} \nu$ for $\mu$. With this knowledge, our remaining input states are of the form

$$
\sum_{x \in \mathbb{F}}\left|w+\nu x+\left(\frac{g}{d}-\frac{c}{d} \nu\right) x^{2}\right\rangle|x\rangle
$$

By subtracting $\frac{g}{d} x^{2}$ from the first register, these become states of the form

$$
\sum_{x \in \mathbb{F}}\left|w+\nu x-\frac{c}{d} \nu x^{2}\right\rangle|x\rangle
$$

The Fourier transform of such a state is

$$
\sum_{y \in \mathbb{F}} \omega^{\operatorname{Tr}(y w)} \sum_{x \in \mathbb{F}} \omega^{\operatorname{Tr}\left(y \nu x-y \frac{c}{d} \nu x^{2}\right)}|y\rangle|x\rangle
$$

which, after measuring the first register, becomes

$$
\sum_{x \in \mathbb{F}} \omega^{\operatorname{Tr}\left(y \nu x-y \frac{c}{d} \nu x^{2}\right)}|x\rangle
$$

With a product of three states of the form (5) with nonzero $y$, we repeat the procedure outlined above. It turns out that we again need to find a nontrivial solution of an equation of the form $y_{1} \delta_{1}^{2}+y_{2} \delta_{2}^{2}+y_{3} \delta_{3}^{2}$ to get the quadratic term of the exponent eliminated and to obtain a proper linear constraint for $\nu$. Having determined $\nu$, we can substitute $\frac{g}{d}-\frac{c}{d} \nu$ for $\mu$. We used six input states to determine the hidden polynomial $f(x)=\nu x+\mu x^{2}$ with high probability.

This procedure can be extended to higher degrees. We will give a formal description in the following subsections. Before that, we briefly outline the method for degree 3, i.e., when $f(x)=\nu x+\mu x^{2}+\kappa x^{3}$. We assume that the characteristic of the base field is greater than 3. In this case, we need four states of the form

$$
\sum_{x \in \mathbb{F}} \omega^{\operatorname{Tr}(y f(x))}|x\rangle
$$

in order to get the cubic term in the exponent of the coefficient eliminated. We obtain such states from the input states by applying the Fourier transform and then a measurement on the first register. To accomplish such an elimination, we have to find a nonzero solution of an equation of the form $y_{1} \delta_{1}^{3}+y_{2} \delta_{2}^{3}+y_{3} \delta_{3}^{3}+y_{4} \delta_{4}^{3}=0$. The result is a similar superposition, with a quadratic exponent. From twelve input states we first collect three 
states with quadratic exponents and from these three states we produce a state with a linear exponent from which we obtain a linear constraint for the unknown coefficients. We then perform a similar procedure using the next twelve input states to obtain a further constraint. Eventually, using 36 input states we will be able to determine all the unknown coefficients with high probability.

\subsection{Statement of the technical generalization}

In this subsection we formulate a technical generalization of the HPGP, which is suitable for recursion. Rather than assuming that the coefficients of the polynomial $f(x)$ are unknown, we assume that they linearly depend on some unknown parameters. This generalization makes it possible to work with polynomials whose coefficients satisfy some already discovered linear constraints. In order to include problems related to certain instances of the hidden subgroup problem, we generalize the problem to tuples of polynomials at the same time.

In the general setting we have level sets of a multidimensional (i.e., vector-valued) function of the form $F(u, x)=u-f(x)$ with $u=\left(u_{1}, \ldots, u_{m}\right)$ and

$$
f(x)=\left(f_{1}(x), \ldots, f_{m}(x)\right) \text { for } f_{i}(x)=\sum_{s=1}^{D} a_{i s}(v) x^{s},
$$

where $a_{i s}(v)$ are known homogeneous linear functions in the unknown $r$-dimensional variable $v=\left(v_{1}, \ldots, v_{r}\right)$. That is, we have

$$
a_{i s}(v)=\sum_{j=1}^{r} a_{i s j} v_{j} .
$$

Theorem 3. Let $m, r$ and $D$ be constants and let $f: \mathbb{F} \rightarrow \mathbb{F}^{m}$ be a function as defined in (6). Then there is a quantum algorithm which, given $O(1)$ states of the form

$$
\sum_{x \in \mathbb{F}}|w+f(x)\rangle|x\rangle
$$

for various unknown vectors $w \in \mathbb{F}^{m}$, determines the unknown parameters $v_{1}, \ldots, v_{r}$ efficiently.

Theorem 1 is the special case of Theorem 3 with $m=1, r=D$, and $a_{1 s}(v)=v_{s}=\nu_{s}$. As a direct consequence, we also obtain the following result regarding a multidimensional generalization of the HPGP to which the hidden subgroup problem in $\mathbb{Z}_{p}^{m} \rtimes \mathbb{Z}_{p}$ can be efficiently reduced (see [2]).

Corollary 4. Let $f(x)=\left(\sum_{s=1}^{D} \nu_{1 s} x^{s}, \ldots, \sum_{s=1}^{D} \nu_{m s} x^{s}\right)$. Then there is a quantum algorithm which, given $O(1)$ states of the form

$$
\sum_{x \in \mathbb{F}}|w+f(x)\rangle|x\rangle
$$

for various unknown $w \in \mathbb{F}^{m}$, determines efficiently the unknown coefficients $\nu_{\text {is }}$ for $i=1, \ldots, m$ and $s=1, \ldots, D$.

Corollary 4 is indeed a special case of Theorem 3 with $r=m D$ and $a_{i s}(v)=$ $v_{(i-1) D+s}=\nu_{i s}$. 


\subsection{A high-level description of the algorithm}

The algorithm for Theorem 1 is organized as a recursion on the number $r$ of the unknown parameters $v_{1}, \ldots, v_{r}$. The recursion (described in Subsection 2.4) is based on eliminating one of the parameters by finding a linear equation for them.

The procedure for finding a linear equation for the parameter starts with producing many states of the form

$$
\sum_{x \in \mathbb{F}} \omega^{\operatorname{Tr}\left(\sum_{j=1}^{r} \sum_{s=1}^{D} v_{j} Y_{j s} x^{s}\right)}|x\rangle
$$

where $Y_{j s}(j=1, \ldots, r, s=1, \ldots, D)$ are elements from $\mathbb{F}$ depending on certain measurements (see Subsection 2.5 for details). During the algorithm we will work with states of the form above, with less and less nonzero coefficients $Y_{j s}$. To shorten the discussion, in this subsection we refer to the polynomial $\sum_{j=1}^{r} \sum_{s=1}^{D} v_{j} Y_{j s} x^{s}$ as the phase of the state.

Assume for simplicity that the characteristic $p$ of our field $F$ is larger than the degree $D$. Then we make small groups of such states. From each group, using a method similar to what is outlined in Subsection 2.1, we fabricate a single state in the phase of which one of the highest degree terms (i.e., $Y_{j D} v_{j} x^{D}$ for some $j$ ) gets eliminated, that is, in the new state the coefficient $Y_{j D}$ becomes zero. From the new states we again form small groups to make states where further high degree terms get eliminated. We proceed this way until we get a state where the phase has linear terms only, that is, a state of the form

$$
\sum_{x \in \mathbb{F}} \omega^{\operatorname{Tr}\left(\sum_{j=1}^{r} v_{j} Y_{j 1} x\right)}|x\rangle
$$

Application of the inverse Fourier transform of $\mathbb{F}$ and a measurement gives then the value of $\sum_{j=1}^{r} v_{j} Y_{j 1}$, which can be used as a linear equation for the parameters $v_{1}, \ldots, v_{r}$.

It turns out that over a field of characteristic $p$ smaller than $D$ the terms of degree $s$, where $s$ is a power of $p$, cannot be eliminated from the phase using a method similar to that of Subsection 2.1. Fortunately, such a method is still applicable to produce a state in the phase of which all the terms whose degree is not a power of $p$ are eliminated, see the first part of Subsection 2.6. The remaining high degree terms are eliminated by using a slightly different technique based on groups of size 2, see the second part of Subsection 2.6 for details.

\subsection{The outer loop}

The algorithm for Theorem 3 uses a recursion by $r$. The main ingredient of the recursion is a procedure (described in the following two subsections), which, using sufficiently many input states, finds with high probability a linear equation

$$
\sum_{j=1}^{r} \alpha_{j} v_{j}=\beta
$$

that is satisfied by the unknown parameters $v_{j}$, where $\alpha_{1}, \ldots, \alpha_{r}, \beta \in \mathbb{F}$ and at least one $\alpha_{i}$ is nonzero. 
Assume without loss of generality that we obtained such an equation with $\alpha_{r} \neq 0$. Then we substitute $v_{r}=\frac{\beta}{\alpha_{r}}-\sum_{j=1}^{r-1} \frac{\alpha_{j}}{\alpha_{r}} v_{j}$. We have

$$
f_{i}(x)=\sum_{s=1}^{D} \sum_{j=1}^{r} a_{i s j} v_{j} x^{s}=\sum_{s=1}^{D} \sum_{j=1}^{r-1}\left(a_{i s j}-\frac{\alpha_{j}}{\alpha_{r}} a_{i s r}\right) v_{j} x^{s}+\frac{\beta}{\alpha_{r}} \sum_{s=1}^{D} a_{i s r} x^{s} .
$$

We apply the recursion to the hidden function $f^{*}=\left(f_{1}^{*}, \ldots, f_{m}^{*}\right)$ with

$$
f_{i}^{*}(x)=f_{i}(x)-\frac{\beta}{\alpha_{r}} \sum_{s=1}^{D} a_{i s r} x^{s} .
$$

Note that the coefficients of $x^{s}$ only depend on the unknown $v_{1}, \ldots, v_{r-1}$ and that the level sets of $u-f^{*}(x)$ and $u-f(x)$ differ only by a shift with

$$
\left(\frac{\beta}{\alpha_{r}} \sum_{s=1}^{D} a_{1 s r} x^{s}, \ldots, \frac{\beta}{\alpha_{r}} \sum_{s=1}^{D} a_{m s r} x^{s}\right) .
$$

This means that if $\left|u_{1}\right\rangle \cdots\left|u_{m}\right\rangle|x\rangle$ belongs to the level set of a certain value $w$ of the function $u-f(x)$ then we subtract $\frac{\beta}{\alpha_{r}} \sum_{s=1}^{D} a_{i s r} x^{s}$ from the $i$ th register for all $i=1, \ldots, m$ and this leads to an element $\left|u_{1}^{*}\right\rangle \cdots\left|u_{m}^{*}\right\rangle|x\rangle$ of the level set of the function $u-f^{*}(x)$ corresponding to $w$. We determine the values $v_{1}, \ldots, v_{r-1}$ by recursion from which $v_{r}$ can be computed using our linear constraint. In the base case of the recursion the main procedure gives us a linear constraint for the only unknown $v_{1}$, which allows us to determine its value easily.

\subsection{The initial stage of the inner procedure}

The level set superpositions for $F(u, x)=u-f(x)$ are states of the form

$$
\sum_{x \in \mathbb{F}}|w+f(x)\rangle|x\rangle=\sum_{x \in \mathbb{F}}\left|w_{1}+\sum_{s=1}^{D} \sum_{j=1}^{r} a_{1 s j} v_{j} x^{s}\right\rangle \cdots\left|w_{m}+\sum_{s=1}^{D} \sum_{j=1}^{r} a_{m s j} v_{j} x^{s}\right\rangle|x\rangle
$$

for various vectors $w=\left(w_{1}, \ldots, w_{m}\right) \in \mathbb{F}^{m}$. We apply the quantum Fourier transform of the field $\mathbb{F}$ independently on all of the first $m$ registers and obtain the state

$$
\sum_{y \in \mathbb{F}^{m}} \sum_{x \in \mathbb{F}} \omega^{\operatorname{Tr}\left(\sum_{i=1}^{m} y_{i} w_{i}+\sum_{i=1}^{m} y_{i} \sum_{j=1}^{r} v_{j} \sum_{s=1}^{D} a_{i s j} x^{s}\right)}|y\rangle|x\rangle
$$

Then we measure $y$ and obtain the state

$$
\omega^{\operatorname{Tr}\left(\sum_{i=1}^{m} y_{i} w_{i}\right)} \sum_{x \in \mathbb{F}} \omega^{\operatorname{Tr}\left(\sum_{i=1}^{m} y_{i} \sum_{j=1}^{r} v_{j} \sum_{s=1}^{D} a_{i s j} x^{s}\right)}|x\rangle
$$

with uniformly random $y=\left(y_{1}, \ldots, y_{m}\right) \in \mathbb{F}^{m}$. After forgetting the global phase, we have

$$
\sum_{x \in \mathbb{F}} \omega^{\operatorname{Tr}\left(\sum_{i=1}^{m} y_{i} \sum_{j=1}^{r} v_{j} \sum_{s=1}^{D} a_{i s j} x^{s}\right)}|x\rangle=\sum_{x \in \mathbb{F}} \omega^{\operatorname{Tr}\left(\sum_{j=1}^{r} v_{j} \sum_{s=1}^{D} Y_{j s} x^{s}\right)}|x\rangle,
$$


where

$$
Y_{j s}=\sum_{i=1}^{m} y_{i} a_{i s j} \text { for } s=1, \ldots, D \text { and } j=1, \ldots, r .
$$

We keep this state only if not all the coefficients $Y_{j s}$ are zero. Provided not all the parameters $a_{i s j}$ are zero, this happens with a probability of at least $\frac{|\mathbb{F}|-1}{|\mathbb{F}|}$. In the following, we use several states of type (8) to obtain similar states, but where the highest-degree term $Y_{j s} x^{s}$ of the phase gets eliminated. We will accomplish this elimination with an iterative method, which is described in the next subsection. Eventually, we obtain a state with linear terms only. Such a state will be used to set up a linear equation for the unknown parameters $v_{j}$.

\subsection{Eliminating high degree terms from the phase}

Here we show how to eliminate the high degree terms from the phase. We consider terms whose degree is a power of $p$ and terms whose degree is not a power of $p$ separately, because the characteristic affects the solvability of equations.

First we describe an iterative procedure which eliminates the terms whose degree is not a power of the characteristic $p$ of $\mathbb{F}$. The iteration is controlled by a single tuple $\left(n_{1}, \ldots, n_{r}\right)$ of integers between 1 and $D$ and we initialize it with

$$
\left(n_{1}, \ldots, n_{r}\right)=(D, \ldots, D) \text {. }
$$

A step of the iteration receives $\ell \leq D+1$ states of the form

$$
\sum_{x \in \mathbb{F}} \omega^{\operatorname{Tr}\left(\sum_{j=1}^{r} v_{j} \sum_{s=1}^{D} Y_{j s} x^{s}\right)}|x\rangle
$$

where we have

$$
Y_{j s}=0 \text { whenever } s>n_{j} \text { and } s \text { is not a power of } p \text {. }
$$

In the case that not all $n_{j}$ are equal to 1 , we define $j_{0}$ to be the smallest index $j$ such that $n_{j}>1$ and the procedure fabricates a state of the form

$$
\sum_{x \in \mathbb{F}} \omega^{\operatorname{Tr}\left(\sum_{j=1}^{r} v_{j} \sum_{s=1}^{D} Y_{j s}^{*} x^{s}\right)}|x\rangle
$$

where not all $Y_{j s}^{*}$ are equal to zero but

$$
Y_{j s}^{*}=0 \text { whenever } s>n_{j} \text { and } s \text { is not a power of } p,
$$

and additionally

$$
Y_{j_{0}, n_{j_{0}}}^{*}=0 \text { if } n_{j_{0}} \text { is not a power of } p \text {. }
$$

The step is trivial if $n_{j_{0}}$ is a power of $p$, or if one of the $\ell$ states, say the $j^{\text {th }}$ state, already satisfies that $n_{j}>1$ and $Y_{j, n_{j}}=0$. In the following, we describe the details of the step for the remaining case. 
The input to the iterative step consists of the elements $Y_{j s}^{(i)} \in \mathbb{F}$ for $i=1, \ldots, \ell$ and $j=1, \ldots, r$ and $s=1, \ldots, D$. We also have the product state

$$
\sum_{\left(x_{1}, \ldots, x_{\ell}\right) \in \mathbb{F}^{\ell}} \omega^{\operatorname{Tr}\left(\sum_{j=1}^{r} v_{j} \sum_{s=1}^{D} \sum_{i=1}^{\ell} Y_{j s}^{(i)} x_{i}^{s}\right)}\left|x_{1}, \ldots, x_{\ell}\right\rangle
$$

where we have

$$
Y_{j s}^{(i)}=0 \text { whenever } s>n_{j} \text { and } s \text { is not a power of } p
$$

but

$$
Y_{j_{0}, n_{j_{0}}}^{(i)} \neq 0 \text { for } i=1, \ldots, \ell .
$$

We assume that $n_{j_{0}}=p^{\beta} b$, where $b$ is an integer that is coprime to $p$ and greater than 1 .

We start with appending $\sum_{x \in \mathbb{F}}|x\rangle$ to the product state $(9)$. This way we obtain

$$
\sum_{\left(x_{1}, \ldots, x_{\ell}\right) \in \mathbb{F}^{\ell}} \sum_{x \in \mathbb{F}} \omega^{\operatorname{Tr}\left(\sum_{j=1}^{r} v_{j} \sum_{s=1}^{D} \sum_{i=1}^{\ell} Y_{j s}^{(i)} x_{i}^{s}\right.}\left|x_{1}, \ldots, x_{\ell}\right\rangle|x\rangle .
$$

Next we choose elements $\delta_{1}, \ldots, \delta_{\ell} \in \mathbb{F}$, which are not all equal to zero, such that

$$
\sum_{i=1}^{\ell}\left(Y_{j_{0}, n_{j_{0}}}^{(i)}\right)^{p^{-\beta}} \delta_{i}^{b}=0
$$

Using $\ell>n_{j_{0}}=p^{\beta} b \geq b$, this can be done in deterministic polynomial time by [7]. For later use we ensure that the tuple $\left(\delta_{1}, \ldots, \delta_{\ell}\right)$ depends only on the ratios between the parameters $Y_{j s}^{(i)}$. This can be done by normalizing the input elements $\left(Y_{j_{0} n_{j_{0}}}^{(i)}\right)^{p^{-\beta}}$ for [7] such that the first nonzero coefficient becomes one.

We subtract $\delta_{i} x$ from the $i$ th register and substitute $x_{i}$ for $x_{i}-\delta_{i} x$ to obtain the state

$$
\sum_{\left(x_{1}, \ldots, x_{\ell}\right) \in \mathbb{F}^{\ell}} \sum_{x \in \mathbb{F}} \omega^{\operatorname{Tr}\left(\sum_{j=1}^{r} v_{j} \sum_{s=1}^{D} \sum_{i=1}^{\ell} Y_{j s}^{(i)}\left(x_{i}+\delta_{i} x\right)^{s}\right)}\left|x_{1}, \ldots, x_{\ell}\right\rangle|x\rangle .
$$

We measure $x_{1}, \ldots, x_{\ell}$ and forget the global phase. Hence, we obtain the state

$$
\sum_{x \in \mathbb{F}} \omega^{\operatorname{Tr}\left(\sum_{j=1}^{r} v_{j} \sum_{k=1}^{D} Y_{j k}^{*} x^{k}\right)}|x\rangle
$$

where

$$
Y_{j k}^{*}=\sum_{s=k}^{D}\left(\begin{array}{l}
s \\
k
\end{array}\right) \sum_{i=1}^{\ell} Y_{j s}^{(i)} x_{i}^{s-k} \delta_{i}^{k} .
$$

Since 10 is a uniform superposition over all choices of $x$ and $x_{1}, \ldots, x_{\ell}$, except with different phases, the measurement produces uniformly random $x_{1}, \ldots, x_{\ell}$.

Note that if $s$ is a power of the characteristic $p$ and if $s>k$ then the integer $\left(\begin{array}{l}s \\ k\end{array}\right)$ is divisible by $p$. Therefore, the terms

$$
\left(\begin{array}{l}
s \\
k
\end{array}\right) \sum_{i=1}^{\ell} Y_{j s}^{(i)} x_{i}^{s-k} \delta_{i}^{k}
$$


are zero. For $s>n_{j}$, which is not a power of $p$, the terms

$$
\left(\begin{array}{l}
s \\
k
\end{array}\right) \sum_{i=1}^{\ell} Y_{j s}^{(i)} x_{i}^{s-k} \delta_{i}^{k}
$$

are zero as well, because the parameters $Y_{j s}^{(i)}$ are all zero. This shows that we have

$$
Y_{j k}^{*}=0 \text { whenever } k>n_{j} \text { and } k \text { is not a power of } p .
$$

We also have

$$
Y_{j_{0}, n_{j_{0}}}^{*}=\sum_{i=1}^{\ell} Y_{j_{0}, n_{j_{0}}}^{(i)} \delta_{i}^{n_{j_{0}}}=\left(\sum_{i=1}^{\ell}\left(Y_{j_{0}, n_{j_{0}}}^{(i)}\right)^{p^{-\beta}} \delta_{i}^{b}\right)^{p^{\beta}}=0
$$

by the choice of the $\delta_{j}$. Furthermore, the equation

$$
Y_{j_{0}, p^{\beta}(b-1)}^{*}=\sum_{s=p^{\beta}(b-1)}^{p^{\beta} b}\left(\begin{array}{c}
s \\
p^{\beta}(b-1)
\end{array}\right) \sum_{i=1}^{\ell} Y_{j_{0}, s^{(i)}}^{\left(x_{i}^{s-p^{\beta}(b-1)} \delta_{i}^{p^{\beta}(b-1)}\right.}
$$

shows that $Y_{j_{0}, p^{\beta}(b-1)}^{*}$ is a polynomial of degree $p^{\beta}$ in the variables $x_{1}, \ldots, x_{\ell}$. The homogeneous part of degree $p^{\beta}$ is

$$
\left(\begin{array}{c}
n_{j_{0}} \\
p^{\beta}(b-1)
\end{array}\right) \sum_{i=1}^{\ell} Y_{j_{0}, n_{j_{0}}}^{(i)} x_{i}^{p^{\beta}} \delta_{i}^{p^{\beta}(b-1)} .
$$

As none of the parameters $Y_{j_{0}, n_{j_{0}}}^{(i)}$ is zero, the coefficients $Y_{j_{0}, n_{j_{0}}}^{(i)} \delta_{i}^{p^{\beta}(b-1)}$ are not all zero. From this we conclude, using the fact that the binomial coefficient

$$
\left(\begin{array}{c}
n_{j_{0}} \\
p^{\beta}(b-1)
\end{array}\right)=\left(\begin{array}{c}
p^{\beta} b \\
p^{\beta}(b-1)
\end{array}\right)=\left(\begin{array}{c}
p^{\beta} b \\
p^{\beta}
\end{array}\right)
$$

is not divisible by $p$ as $1<b<p$, that $Y_{j_{0}, p^{\beta}(b-1)}^{*}$ is not identically zero when considered as a polynomial in the variables $x_{1}, \ldots, x_{\ell}$. As the measurements give us uniformly random values $x_{1}, \ldots, x_{\ell}$, we know by the Schwartz-Zippel lemma that $Y_{j_{0}, p^{\beta}(b-1)}^{*}$ will be nonzero with a probability of at least

$$
\frac{|\mathbb{F}|-p^{\beta}}{|\mathbb{F}|} \geq \frac{|\mathbb{F}|-D}{|\mathbb{F}|}
$$

This shows that not all the new coefficients $Y_{j s}^{*}$ for $s \leq n_{j}$ will be zero with a probability of at least $\frac{|\mathbb{F}|-D}{|\mathbb{F}|}$, because $Y_{j_{0}, p^{\beta}(b-1)}^{*}$ is one of these coefficients. With high likelyhood, we have produced a state of the form 11 for a set of new coefficients $Y_{j s}^{*}$ that are known linear polynomials in the original coefficients $Y_{j s}^{(i)}$.

After eliminating the terms whose degrees are not a power of $p$, we now explain how to deal, in the remaining rounds of the iteration, with terms whose degrees are a power of $p$. Our intention is the following. From several states of the form

$$
\sum_{x \in \mathbb{F}} \omega^{\operatorname{Tr}\left(\sum_{j=1}^{r} v_{j} \sum_{t=0}^{d} Z_{j t} x^{p^{t}}\right)}|x\rangle,
$$


where $d=\left\lfloor\log _{p} D\right\rfloor$ and not all of the coefficients $Z_{j t}$ are equal to zero, we produce a state that has only linear terms. Now the iteration is controlled by a single tuple $\left(n_{1}, \ldots, n_{r}\right)$ of integers between 0 and $d$, which are not all equal to zero, and the iteration starts with the tuple

$$
\left(n_{1}, \ldots, n_{r}\right)=(d, \ldots, d) .
$$

A step of the iteration receives the coefficients $Z_{j t}^{(i)}$ for $j=1, \ldots, r$ and $t=0, \ldots, d$ and $i=1,2$ along with the two states

$$
\sum_{x_{i} \in \mathbb{F}} \omega^{\operatorname{Tr}\left(\sum_{j=1}^{r} v_{j} \sum_{t=0}^{d} Z_{j t}^{(i)} x_{i}^{p^{t}}\right)}\left|x_{i}\right\rangle
$$

such that for both $i=1,2$ the coefficients $Z_{j t}^{(i)}$ are not all zero but $Z_{j t}^{(i)}=0$ whenever $t>n_{j}$. Let $j_{0}$ be the smallest integer $j$ such that $n_{j}>0$. We simply pass the appropriate state to the next round if either $Z_{j_{0}, n_{j_{0}}}^{(1)}$ or $Z_{j_{0}, n_{j_{0}}}^{(2)}$ is zero.

Otherwise let us assume first that there exist two pairs $\left(t_{1}, j_{1}\right)$ and $\left(t_{2}, j_{2}\right)$ with $t_{1} \neq t_{2}$ such that $Z_{j_{1}, t_{1}}^{(1)} \neq 0$ and $Z_{j_{2}, t_{2}}^{(2)} \neq 0$. Then we abort if there is an element $z \in \mathbb{F}$ such that $Z_{j t}^{(2)}=z^{p^{t}} Z_{j t}^{(1)}$ for all $j=1, \ldots, r$ and for all $t=0, \ldots, d$. Otherwise we append $\sum_{x \in \mathbb{F}}|x\rangle$ to the product state

$$
\sum_{\left(x_{1}, x_{2}\right) \in \mathbb{F}^{2}} \omega^{\operatorname{Tr}\left(\sum_{j=1}^{r} v_{j} \sum_{t=0}^{d}\left(Z_{j t}^{(1)} x_{1}^{p^{t}}+Z_{j t}^{(2)} x_{2}^{p^{t}}\right)\right)}\left|x_{1}, x_{2}\right\rangle
$$

and we obtain

$$
\sum_{x \in \mathbb{F}} \sum_{\left(x_{1}, x_{2}\right) \in \mathbb{F}^{2}} \omega^{\operatorname{Tr}\left(\sum_{j=1}^{r} v_{j} \sum_{t=0}^{d}\left(Z_{j t}^{(1)} x_{1}^{p^{t}}+Z_{j t}^{(2)} x_{2}^{p^{t}}\right)\right)}\left|x_{1}, x_{2}\right\rangle|x\rangle
$$

Then we set

$$
\delta_{1}=1 \text { and } \delta_{2}=-\left(\frac{Z_{j_{0}, n_{j_{0}}}^{(1)}}{Z_{j_{0}, n_{j_{0}}}^{(2)}}\right)^{p^{-n_{j_{0}}}}
$$

and we subtract $\delta_{i} x$ from the $i$ th register. After substituting $x_{i}$ for $x_{i}-\delta_{i} x$ we obtain the state

$$
\sum_{x \in \mathbb{F}} \sum_{\left(x_{1}, x_{2}\right) \in \mathbb{F}^{2}} \omega^{\operatorname{Tr}\left(\sum_{j=1}^{r} v_{j} \sum_{t=0}^{d}\left(Z_{j t}^{(1)}\left(x_{1}+\delta_{1} x\right)^{p^{t}}+Z_{j t}^{(2)}\left(x_{2}+\delta_{2} x\right)^{p^{t}}\right)\right)}\left|x_{1}, x_{2}\right\rangle|x\rangle .
$$

We measure $x_{1}, x_{2}$ and after forgetting the global phase we obtain the state

$$
\sum_{x \in \mathbb{F}} \omega^{\operatorname{Tr}\left(\sum_{j=1}^{r} v_{j} \sum_{t=0}^{d} Z_{j t}^{*} x^{p^{t}}\right)}|x\rangle,
$$

where we have

$$
Z_{j t}^{*}=Z_{j t}^{(1)} \delta_{1}^{p^{t}}+Z_{j t}^{(2)} \delta_{2}^{p^{t}}
$$

By the choice of $\delta_{1}$ and $\delta_{2}$, we have $Z_{j_{0}, n_{j_{0}}}^{*}=0$, and by the assumption

$$
Z_{j t}^{(2)} \neq-Z_{j t}^{(1)}\left(\frac{\delta_{1}}{\delta_{2}}\right)^{p^{t}}
$$


for some $j$ and $t$ not all $Z_{j t}^{*}$ are equal to zero. Again, $\delta_{1}$ and $\delta_{2}$ depend only on the ratios between the parameters $Z_{j t}^{(i)}$.

If all $Z_{j t}^{(1)}$ are zero except for $t=n_{j_{0}}$ then we replace $x_{1}$ with $x_{1}^{p^{-n_{j}}}$ and finish the iteration with the state

$$
\sum_{x_{1} \in \mathbb{F}} \omega^{\operatorname{Tr}\left(\sum_{j=1}^{r} v_{j} Z_{j, n_{j}}^{(1)} x_{1}\right)}\left|x_{1}\right\rangle
$$

and the inverse Fourier transform gives us the sum

$$
\sum_{j=1}^{r} v_{j} Z_{j, n_{j_{0}}}^{(1)}
$$

for the unknown $v_{1}, \ldots, v_{r}$.

The iterative procedure above, starting with $L=O(1)$ states of the form (8) with uniformly random $y_{1}, \ldots, y_{m}$, constructs a state of the form

$$
\sum_{x \in \mathbb{F}} \omega^{\operatorname{Tr}\left(\sum_{j=1}^{r} \alpha_{j} v_{j} x\right)}|x\rangle
$$

where not all of the $\alpha_{j}$ are zero, with high success probability in time polylog $(|\mathbb{F}|)$. We apply the inverse of the quantum Fourier transform of $\mathbb{F}$ to the state 12 and obtain the state

$$
\left|\sum_{j=1}^{n} \alpha_{j} v_{j}\right\rangle .
$$

When we measure this state and denote the result by $\beta$, then we have a linear constraint of the form (7) for the unknown $v_{j}$.

The probability of abortion, i.e., there is a $z \in \mathbb{F}$ with $Z_{j t}^{(2)}=z^{p^{t}} Z_{j t}^{(1)}$ for all $j$ and $t$, can be estimated as follows. First, assume that for a run of the iteration to compute the state with coefficients $Z_{j t}^{(2)}$ we have tuples $\left(y_{1}^{(i)}, \ldots, y_{m}^{(i)}\right)$ with $i=1, \ldots, L$ as measurement results in the beginning. Then for a $\gamma \in \mathbb{F} \backslash\{0\}$ the iteration for the measurement results $\left(\gamma y_{1}^{(i)}, \ldots, \gamma y_{m}^{(i)}\right)$ takes the same course and we obtain the coefficients $\gamma Z_{j t}^{(2)}$, because all $Y_{j s}^{(i)}$ and $Z_{j t}^{(i)}$ are just homogeneous linear combinations. When we have $Z_{j t}^{(2)}=z^{p^{t}} Z_{j t}^{(1)}$ for all $j$ and $t$, then for $\gamma \neq 0$ we would have $\gamma Z_{j t}^{(2)}=z^{p^{t}} Z_{j t}^{(1)}$ and this cannot hold for all $t$ when $\gamma \neq 1$. Therefore, when we consider a fixed first state $Z_{j t}^{(1)}$, then for each measurement result for the second collection of states that leads to an abortion because of $Z_{j t}^{(2)}=z^{p^{t}} Z_{j t}^{(1)}$, there are at least $|\mathbb{F}|-2$ possibilities of other measurement results that do not lead to abortion. The normalization in both of the iteration steps ensures that for every $w \in \mathbb{F}$ the probability of obtaining a state with $\gamma Z_{j t}^{(2)}$ instead of $Z_{j t}^{(2)}$ for every $t$ and $j$ are the same for every $\gamma \in \mathbb{F} \backslash\{0\}$. Therefore, if $Z_{j_{1}, t_{1}}^{(1)}$ and $Z_{j_{2}, t_{2}}^{(2)}$ are nonzero for two pairs $\left(j_{1}, t_{1}\right)$ and $\left(j_{2}, t_{2}\right)$, the conditional property of having $Z_{j t}^{(2)}=z^{p^{t}} Z_{j t}^{(1)}$ is at most $\frac{1}{|\mathbb{F}|-1}$.

This finishes the description of the algorithm for Theorem 3. 


\section{Application: Special hidden polynomials}

In this section we prove Theorem 2. To simplify the notation, we define the cardinality of the level set of the function $F(x, y)=g(y)-f(x)$ corresponding to $w \in \mathbb{F}$ to be

$$
M_{F}(w)=\#\left\{(x, y) \in \mathbb{F}^{2}: g(y)-f(x)=w\right\}
$$

and in a similar manner

$$
m_{g}(w)=\#\{y \in \mathbb{F}: g(y)=w\}
$$

to be the cardinality of the level set of $g(y)$ corresponding to $w \in \mathbb{F}$. We trivially have that

$$
\sum_{w \in \mathbb{F}} M_{F}(w)=|\mathbb{F}|^{2}
$$

and that

$$
\sum_{x \in \mathbb{F}} m_{g}(f(x)+w)=M_{F}(w)
$$

for every $w \in \mathbb{F}$. The quantum procedure starts with the uniform superposition

$$
\frac{1}{|\mathbb{F}|} \sum_{x, y \in \mathbb{F}}|y\rangle|x\rangle|0\rangle
$$

and we apply the oracle to produce the state

$$
\frac{1}{|\mathbb{F}|} \sum_{x, y \in \mathbb{F}}|y\rangle|x\rangle|\mathcal{E}(F(x, y))\rangle
$$

Then we measure the third register and we obtain the result $\mathcal{E}(w)$ for a $w \in \mathbb{F}$ with probability $M_{F}(w) /|\mathbb{F}|^{2}$. The resulting state of the first two registers is

$$
\left|\Phi_{w}\right\rangle=\frac{1}{\sqrt{M_{F}(w)}} \sum_{\left\{(x, y) \in \mathbb{F}^{2}: g(y)-f(x)=w\right\}}|y\rangle|x\rangle .
$$

Note that this state is similar to the level set superposition of the function $y-f(x)$ corresponding to the value $w$. To exploit this connection, we make use of the unitary map $\mathcal{U}_{g}$ which maps $|z\rangle$ to $\frac{1}{\sqrt{m_{g}(z)}} \sum_{y: g(y)=z}|y\rangle$ for $z \in \mathbb{F}$. The case that $g(y)=z$ has no solution cannot occur in our algorithm and therefore we set the result of $\mathcal{U}_{g}$ to a special state in this case. We can implement $\mathcal{U}_{g}$ as follows.

1. Compute $S_{z}=\{y: g(y)=z\}$ in an ancilla using Berlekamp's root finding algorithm [8].

2. Produce the uniform superposition $\left|S_{z}\right\rangle=\frac{1}{\sqrt{m_{g}(z)}} \sum_{y \in S_{z}}|y\rangle$ in another ancilla.

3. Erase the first ancilla by undoing the first step.

4. Swap $|z\rangle$ with $\left|S_{z}\right\rangle$.

5. Erase the ancilla holding $|z\rangle$ by evaluating $g$ on $\left|S_{z}\right\rangle$. 
We apply $\mathcal{U}_{g}$ to the first register of $\left|\Phi_{w}\right\rangle$ and obtain the state

$$
\left|\Psi_{w}\right\rangle=\frac{1}{\sqrt{M_{F}(w)}} \sum_{x \in \mathbb{F}} \sqrt{m_{g}(f(x)+w)}|w+f(x)\rangle|x\rangle .
$$

Let $\left|\Lambda_{w}\right\rangle$ stand for the state corresponding to the level set $\{x \in \mathbb{F}: u-f(x)=w\}$ of the function $u-f(x)$, that is,

$$
\left|\Lambda_{w}\right\rangle=\frac{1}{\sqrt{|\mathbb{F}|}} \sum_{x \in \mathbb{F}}|w+f(x)\rangle|x\rangle .
$$

Then the scalar product of $\left|\Psi_{w}\right\rangle$ with $\left|\Lambda_{w}\right\rangle$ is

$$
\left\langle\Psi_{w} \mid \Lambda_{w}\right\rangle=\frac{1}{\sqrt{M_{F}(w)|\mathbb{F}|}} \sum_{x \in \mathbb{F}} \sqrt{m_{g}(f(x)+w)} .
$$

We apply the inequality $m_{g}(f(x)+w) \leq \operatorname{deg}(g)=D^{\prime}$ to obtain

$$
\left\langle\Psi_{w} \mid \Lambda_{w}\right\rangle \geq \frac{1}{\sqrt{M_{F}(w)|\mathbb{F}|}} \sum_{x \in \mathbb{F}} \frac{1}{\sqrt{D^{\prime}}} m_{g}(f(x)+w)=\frac{\sqrt{M_{F}(w)}}{\sqrt{D^{\prime}|\mathbb{F}|}} .
$$

The expected value of $M_{F}(w)$ is

$$
\sum_{w \in \mathbb{F}} \frac{M_{F}(w)}{|\mathbb{F}|^{2}} M_{F}(w) \geq \frac{1}{|\mathbb{F}|}\left(\frac{1}{|\mathbb{F}|} \sum_{w \in \mathbb{F}} M_{F}(w)\right)^{2}=\frac{1}{|\mathbb{F}|}\left(\frac{1}{|\mathbb{F}|}|\mathbb{F}|^{2}\right)^{2}=|\mathbb{F}|
$$

As the maximum possible value for $M_{F}(w)$ is $D^{\prime}|\mathbb{F}|$ and as $D^{\prime}$ is a constant, we have that $M_{F}(w)$ is at least $\frac{1}{2}|\mathbb{F}|$ with a probability that is lower bounded by a positive constant. This implies that the scalar product of $\left|\Psi_{w}\right\rangle$ with $\left|\Lambda_{w}\right\rangle$ is also at least another positive constant. Therefore, if we apply the algorithm of Theorem 1 to $O(1)$ states of the form $\left|\Phi_{w}\right\rangle$ instead of $\left|\Lambda_{w}\right\rangle$, we still have a probability, which is lower bounded by a positive constant, that the algorithm determines the coefficients of $f(x)$ correctly.

Using the above procedure we obtain a guess $f_{0}(x)$ for $f(x)$. We can test correctness of such a guess using the oracle as follows. As $\#\left\{(x, y) \in \mathbb{F}^{2}: g(y)-f_{0}(x)=w\right\}$ is $|\mathbb{F}|$ on average and as the maximum is $D^{\prime}|\mathbb{F}|$, for a uniformly random $w$ this number will be at least $|\mathbb{F}| / 2$ with a probability of at least $\frac{1}{2 D^{\prime}}$. For such a $w$, at least for $\frac{|\mathbb{F}|}{2 D}$ values $x$, the equation $g(y)=f_{0}(x)+w$ has at least one solution $y$ which can be obtained by Berlekamp's root finding algorithm. Using this strategy, we find in polylog $(|\mathbb{F}|)$ time with high probability an element $w \in \mathbb{F}$ and $D+1$ pairs $\left(x_{1}, y_{1}\right), \ldots,\left(x_{D+1}, y_{D+1}\right)$ from $\mathbb{F}^{2}$ such that $x_{i} \neq x_{j}$ holds whenever $i \neq j$ and $f_{0}\left(x_{i}\right)-g\left(y_{i}\right)=w$ for $i=1, \ldots, D+1$. We call the oracle for the pairs $\left(x_{i}, y_{i}\right)$ and check if it returns the same value for all $i=1, \ldots, D+1$. If it does not then it is impossible that $f$ and $f_{0}$ are the same up to constant term. However, if it does then we have $f\left(x_{i}\right)=g\left(y_{i}\right)+w^{\prime}$ for $i=1, \ldots, D+1$ and for some $w^{\prime} \in \mathbb{F}$, whence $f\left(x_{i}\right)=f_{0}\left(x_{i}\right)+w^{\prime}-w$ for $i=1, \ldots, D+1$. From this, $f(x)=f_{0}(x)+w^{\prime}-w$ follows for every $x \in \mathbb{F}$ because $f$ has degree at most $D$. This completes the proof of Theorem 2 , 


\section{Acknowledgments}

Most of this work was conducted at the Centre for Quantum Technologies (CQT) in Singapore, and partially funded by the Singapore Ministry of Education and the National Research Foundation, also through the Tier 3 Grant "Random numbers from quantum processes". Research partially supported by the Natural Sciences and Engineering Research Council of Canada, the European Commission IST STREP projects Quantum Computer Science (QCS) 255961 and Quantum Algorithms (QALGO) 600700, by the French ANR Blanc program under contract ANR-12-BS02-005 (RDAM project), and by the Hungarian Scientific Research Fund (OTKA), Grants NK105645 and K77476.

\section{References}

\section{References}

[1] A. Childs, L. Schulman and U. Vazirani (2007), Quantum Algorithms for Hidden Nonlinear Structures, In: Proc. 48th IEEE Symposium on Foundations of Computer Science (FOCS), pp. 39-404. arxiv:0705.2784, doi:10.1109/FOCS.2007.18.

[2] T. Decker, G. Ivanyos, M. Santha and P. Wocjan (2011), Hidden symmetry subgroup problems, Technical report arxiv:1107.2189 [quant-ph]; SIAM J. Comput, to appear. Technical report, 2011. arxiv:1107.2189.

[3] T. Decker, J. Draisma and P. Wocjan (2009), Quantum algorithm for identifying hidden polynomial function graphs, Quantum Inform. Comput., Vol. 9, pp. 0215 0230. arxiv:0706.1219.

[4] D. Bacon, A. Childs and W. van Dam (2005), From optimal measurement to efficient quantum algorithms for the hidden subgroup problem over semidirect product groups, In: Proc. 46th IEEE Symposium on Foundations of Computer Science (FOCS), pp. 469-478. arxiv:0504083, doi:10.1109/SFCS.2005.38.

[5] W. van Dam, S. Hallgren and L. Ip (2006), Quantum algorithms for some hidden shift problems, SIAM J. Comput., Vol. 36, p. 763-778. arxiv:0211140, doi:10. 1137/S009753970343141X.

[6] G. Ivanyos, L. Sanselme and M. Santha (2007), An efficient quantum algorithm for the hidden subgroup problem in extraspecial groups, In: Proc. STACS 2007 (LNCS Vol. 4393), pp. 586-597. arxiv:0701235, doi:10.1007/978-3-540-70918-3_50.

[7] C. E. van de Woestijne (2006), Deterministic equation solving over finite fields. $\mathrm{PhD}$ thesis, Universiteit Leiden, 2006. hdl:1887/4392.

[8] E. R. Berlekamp (1968), Algebraic Coding Theory, McGraw-Hill, (New York). 